DR. KIAN FAN CHUNG (Orcid ID : 0000-0001-7101-1426)

Article type : Letter to the Editor

\title{
Type 2-low asthma phenotypes by integration of sputum transcriptomics and serum proteomics
}

To the Editor

Asthma is a complex heterogeneous disease that presents with varying degrees of severity. Analysis of the transcriptome of sputum cells from asthma patients have defined one T2-high and two T2-low clusters (1). To obtain a better granularity of the T2-low clusters, we have performed a combined analysis of sputum transcriptomics with serum proteomics using an integrative machine learning work-flow (Supplementary Figure S1). We studied 104 patients with asthma and 16 normal healthy controls from the UBIOPRED cohort (2) and re-analysed the 508 differentially-expressed genes (DEGs) of sputum cell transcriptomics between high eosinophil and low eosinophil counts (1) by applying consensus clustering and finite Gaussian Mixture Model (GMM) clustering (Supplementary Table S1; Supplementary Methods and Results). We identified four optimal clusters (TAC*1, TAC*2, TAC*3a and TAC*3b) (Supplementary Figure S3), in agreement with our previous clustering (1) where TAC*3a and TAC*3b were combined. Similar clusterings were generated using complete linkage hierarchical agglomerative clustering, K-means and partitioning around medoids. TAC*1 patients were more severe asthmatics with a greater use of oral corticosteroid therapy, more nasal polyps and a higher level of sputum eosinophils compared to TAC*2 (Supplementary Table S2; Supplementary Figure S4). $\mathrm{TAC}^{*} 3 \mathrm{a}$ included more severe asthmatics on oral corticosteroid therapy, with a higher sputum neutrophilia, serum C-reactive protein levels compared to TAC*3b patients. Metacore pathway analysis (http://metacore.com) using overrepresentation analysis and shrunken centroid method (3) indicated key-regulated

This article has been accepted for publication and undergone full peer review but has not been through the copyediting, typesetting, pagination and proofreading process, which may lead to differences between this version and the Version of Record. Please cite this article as doi:

10.1111/ALL.14573

This article is protected by copyright. All rights reserved 
immune pathways that distinguished the $4 \mathrm{TAC}^{*} \mathrm{~s}$. Gene set variation analysis (GSVA) (4), an enrichment analysis of specific gene-signatures shown in Supplementary Table S3, indicated that TAC*1 had the highest expression score (ES) for the T2-high gene signature while TAC*2 showed up-regulation of cell-killing pathway via CD8+ T-cells, NK cells and macrophages, inflammasome activation and toll-like receptor (TLR) pathogensensing pathways. While $\mathrm{TAC}^{*} 3 \mathrm{a}$ showed up-regulation of pathways similar to $\mathrm{TAC}^{\star} 2$, $T A C^{*} 3 b$ showed attenuation of TLR sensor pathway activation with down-regulation of cellular responses in asthma, malignancy and autoimmunity compared to TAC*3a.

GSVA indicated that the IL13 gene signature ES was highest in TAC*1 while the ILC1 signature was highly enriched in TAC*2 followed by TAC*1 and also exhibited higher ES scores in TAC*3a compared to TAC*3b (Figure 1, Supplementary Figures S5 \& S6). The inflammasome pathway and activated neutrophil ES scores were highly enriched in TAC*2 and TAC*3a compared to TAC*3b. In contrast, the KEGG oxidative phosphorylation pathway showed higher ES score in TAC*3b compared to TAC*3a, with lower enrichment in both $\mathrm{TAC}^{*} 1$ and $\mathrm{TAC}^{*} 2$. The enrichment of activated polarized human peripheral blood Th17 cell ES was enhanced in TAC*3b, but there was no difference for ILC3 cell ES across the TAC*s while ILC2 cell ES were enriched in TAC*1 compared to other TAC*S (Supplementary Figures S5 \& S6). These clusters were validated in the independent ADEPT (5) and Australian (6) asthma cohorts by comparing the ESs of the signature genes of each of the 4 TAC*s across the 3 cohorts. The distribution of the ESs was not significantly different (Supplementary Figure S7).

Sputum transcriptomics and serum proteomics from 92 asthmatic subjects using SomaScan technology (SomaLogic, Boulder, CO, USA) (7) and shot-gun proteomics using liquid chromatography-mass spectrometry (LC-MS/MS) (8) were then integrated using Similarity Network Fusion (SNF) (Supplementary Figure S2). SNF is an efficient integrative method for different data-sets that constructs similarity networks based on each data-set and then integrates these networks into a single network that represents all underlying data-types (9). By pre-selecting the number of clusters to 4 , we reproduced these 4 TAC*s with a high degree of concordance $(81 \%)$ when fusing with proteomic data, indicating the robustness of the SNF approach. Allowing the data to freely cluster, we identified 9 proteomic- and transcriptomic-associated clusters (PTACs) derived from the 4 TAC* clusters (Figure 2; Supplementary Figures S8 \& S9). PTAC2a $(n=14)$ showed 
autoimmune pathways related to multiple sclerosis and systemic lupus erythematosus (SLE) while PTAC2b $(n=7)$ enrichment of TLR pathways, type I interferon and inflammasome activation, responses associated with infective pathogens. PTAC2a subjects had evidence for macrophage, NK and TC1 cell activation with enhancement of IL-2, IL-6 and apoptotic pathways whilst PTAC2b was associated with enhanced type I IFN and inflammasome activation. PTAC2a patients had the highest percentage of patients with severe asthma who were smokers and had a more frequent history of pneumonia and prevalence of atopy, compared to PTAC2b. PTAC*3a1, PTAC*3a2 and PTAC*3a3 participants showed clear reduction in T2 pathways compared to TAC*1. Furthermore, PTAC3a1 $(n=14)$ and PTAC3a2 $(n=6)$ participants were differentiated with respect to severe asthma, prevalence of nasal polyps, smoking history, prevalence of eczema and a history of pneumonia with PTAC3a2 with the highest percentage of severe asthma who were smokers or ex-smokers, with a more frequent history of pneumonia, prevalence of atopy and nasal polyps, compared to PTAC3a1 subjects. PTAC3b1 $(n=10)$, PTAC3b2 $(n=6)$ and PTAC3b3 $(n=8)$ had different levels of blood leukocytes, blood neutrophils, and C-reactive protein, and those on oral corticosteroids. TAC*3 primarily comprised of subjects associated with OXPHOS and ageing pathways but their delineation remained less clear than PTAC2. Reduced OXPHOS compared with healthy controls in TAC1, TAC2 and TAC3a indicate a potential switch to glycolytic processes but TAC3b with significantly increased OXPHOS respond to distinct mitochondrial or metabolic treatment modalities (Supplementary Figure S10). However, this analysis is limited by the small number of subjects within the PTAC groups and larger validation studies will be required to ascertain the identification of distinct pathways. Interestingly, TAC1 remains undivided, indicating that serum proteomics did not bring any factors that allowed for subclusters.

We have defined 9 molecular clusters, distinguished by different combinations of ES of distinct pathways that included T2-high and T2-low pathways, and associated with differences in clinical and inflammatory characteristics. T2-low asthma is a heterogeneous condition of 8 clusters compared to the homogeneity of the single T2-high cluster. Thus, the addition of the 2 proteomic platforms to the analysis has allowed us to provide a greater granularity of the T2-low clusters. The existence of multiple T2-low asthma phenotypes is challenging with requirements for specific biomarkers and different targeted therapies compared to the single T2-high cluster. 
Another important finding in this analysis is the repartition of various potential pathways involved in asthma in the subclusters generated in this study, as assessed by GSVA. Although the IL-13-T2-signature that we used indicated the highest expression in TAC1, it was also seen in individuals in TAC2. Other pathways that could potentially drive asthma pathobiology include ILC1 in TAC1 and TAC2, inflammasome, neutrophil and IL17 activation in TAC2, OXPHOS in TAC3a and TAC3b, and IFNy activation in TAC2 or TAC3a.

Finally, one limitation of our study is the unknown effects of corticosteroid therapy particularly oral prednisolone on different inflammatory pathways such as the T2-high, thus representing a confounding factor. There is also a degree of corticosteroid insensitivity since that T2-high TAC1 cluster had the highest level of eosinophilia despite $58 \%$ of the group being on daily oral corticosteroids (OCS). Alternatively, this might represent the most severe patients with asthma. The effect of this therapy on molecular clustering can only be determined by studying the effect of OCS in this population.

1169 words

This article is protected by copyright. All rights reserved 
Nazanin Zounemat Kermani ${ }^{1}$, Mansoor Saqi ${ }^{1}$, Paul Agapow ${ }^{1}$,Stelios Pavlidis ${ }^{1}$, Chihhsi Kuo ${ }^{1,2}$, Kai Sen $\operatorname{Tan}^{10}$, Sharon Mumby², Kai Sun ${ }^{1}$, Matthew Loza ${ }^{3}$, Frederic Baribaud ${ }^{3}$, Ana R Sousa ${ }^{4}$, John Riley ${ }^{4}$, Asa M. Wheelock ${ }^{5}$, Craig E. Wheelock ${ }^{5}$, Bertrand De Meulder $^{6}$, Jim Schofield 7 , Stephany Sánchez-Ovando ${ }^{8}$, Jodie Louise Simpson ${ }^{8}$, Katherine Joanne Baines ${ }^{8}$, Peter A Wark ${ }^{8}$, Charles Auffray ${ }^{6}$, Sven-Erik Dahlen ${ }^{5}$, Peter J Sterk $^{9}$, Ratko Djukanovic ${ }^{7}$, *Ian M. Adcock1, 2, Yi-ke Guo ${ }^{1}$, *Kian Fan Chung ${ }^{1,2}$ on behalf of U-BIOPRED project team

1Department of Computing \& Data Science Institute, Imperial College London, United Kingdom; ${ }^{2}$ National Heart \& Lung Institute, Imperial College London; ${ }^{3}$ Janssen Research and Development, High Wycombe, Buckinghamshire, United Kingdom; ${ }^{4}$ Respiratory Therapeutic Unit, GSK, Stockley Park, UK; ${ }^{5}$ Dept of Medicine Solna \& Center for Molecular Medicine \& Department of Medical Biochemistry and Biophysics, Karolinska Institute, Stockholm, Sweden; ${ }^{6} E$ uropean Institute for Systems Biology and Medicine, CNRS-ENS-UCBL-INSERM, Lyon, France; ${ }^{7}$ Faculty of Medicine, Southampton University, Southampton, UK and NIHR Southampton Respiratory Biomedical Research Unit, University Hospital Southampton, Southampton, UK; ${ }^{8}$ Priority Research Centre for Healthy Lungs, Faculty of Health and Medicine, University of Newcastle, NSW Australia; ${ }^{9}$ Amsterdam University Medical Centers, University of Amsterdam, Amsterdam, Netherlands, ${ }^{10}$ Department of Otolaryngology, National University of Singapore, Singapore.

\section{Corresponding author}

Professor K F Chung, National Heart \& Lung Institute, Imperial College London, Dovehouse St, London SW3 6LY, UK ～f.chung@imperial.ac.uk

\section{Funding sources:}

U-BIOPRED was supported by an Innovative Medicines Initiative Joint Undertaking (No.115010), resources from the European Union's Seventh Framework Programme (FP7/2007-2013) and EFPIA companies' in-kind contribution (www.imi.europa.eu). 


\section{Conflict of interest}

Ratko Djukanović has consulted and presented at symposia organised by TEVA, Novartis, GlaxoSmithKline and AstraZeneca, has shares in and consults for Synairgen; Dr. Asa Wheelock report remuneration from AstraZenica and Harvard Medical School for speaking engagements on SNF-clustering in COPD.; Charles Auffray reports grants from Innovative Medicine Initiative; Kian Fan Chung has received honoraria for participating in Advisory Board meetings of the pharmaceutical industry regarding treatments for asthma and chronic obstructive pulmonary disease and has also been remunerated for speaking engagements; lan Adcock has received grants from Advisory Board meetings with pharmaceutical companies GSK, A-Z, Novartis, Boehringer Ingelheim and Vectura, and grants on asthma and COPD from Pfizer, GSK, MRC, EU, BI and IMI; Peter Sterk reports grants from IMI Innovative Medicines Initiative, during the conduct of the study; Matthew Loza and Frederic Baribaud are Employees and Shareholders of Janssen Research and Development, a Johnson and Johnson company; John Riley and Ana R Sousa are employees of GSK; the rest of the authors have nothing to disclose.

\section{Contribution}

NZK, MS, SP, PA, KST, CK and AV have contributed to the analysis of the data at various stages; KS, ML, FB, ARS \& SM have participated in data curation and quality control; JR, AW, CW, BDM, YKG and DB have contributed to the analytical plan and methods; RB, CA, PJS, SED, IMA and KFC to discussion of the overall strategy and plan; JLS, KJB and PAW provided the data from Australian cohort; NZK, IMA and KFC to the overall direction of this study and writing of the paper.

Supplementary file: Supplementary Methods and Results; 3 Supplementary Tables, and 9 Supplementary Figures.

\section{Key words:}

asthma, bioinformatics, endotypes, systems biology, precision medicine. 


\section{Figures:}
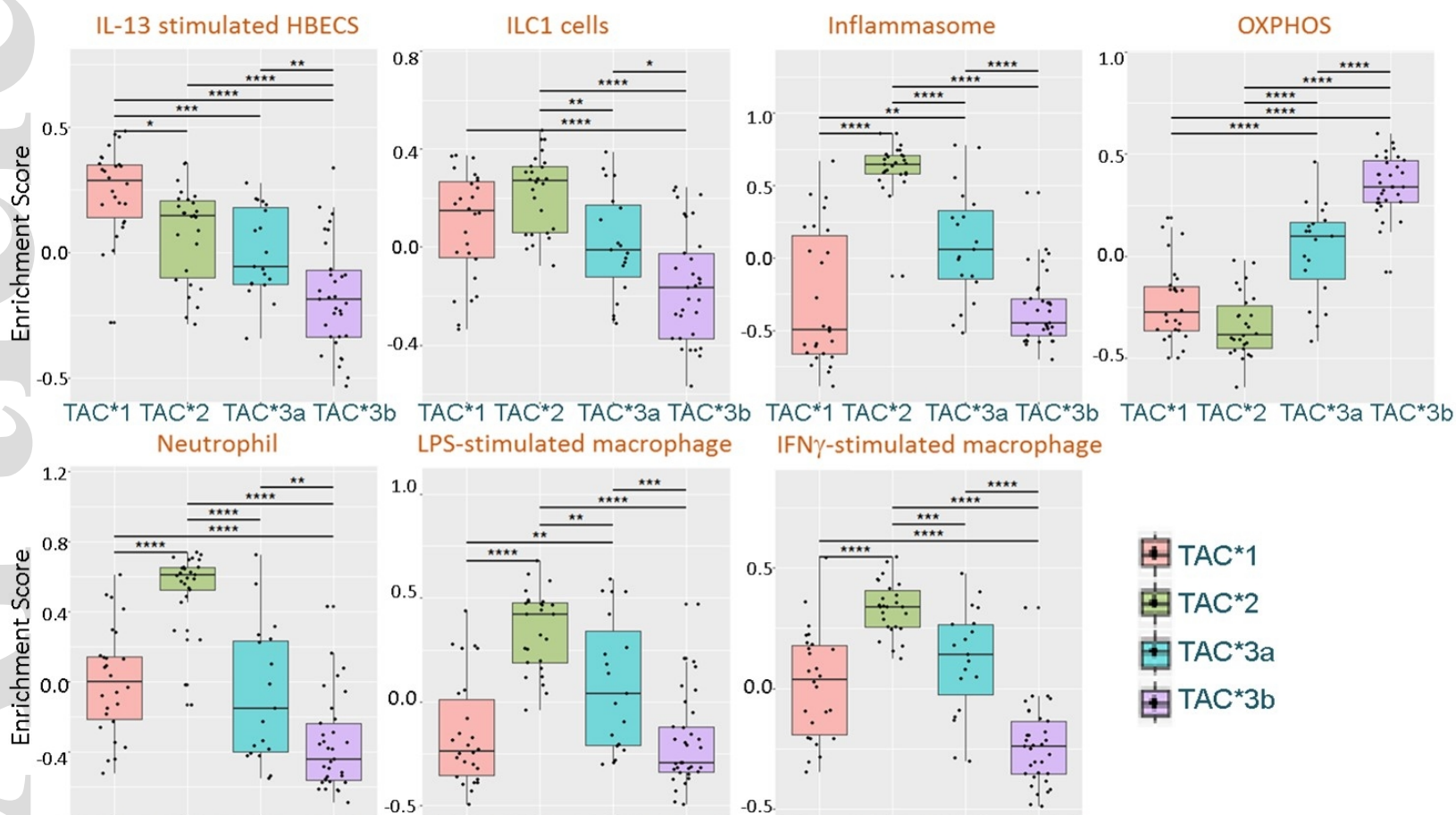

$\mathrm{TAC}^{*} 1 \mathrm{TAC}^{*} 2 \mathrm{TAC} * 3 a \mathrm{TAC} * 3 \mathrm{~b}$ TAC*1 TAC*2 TAC*3a TAC*3b TAC*1 TAC*2 TAC*3a TAC*3b

Figure 1. Gene set variation analysis. Dot plot enrichment scores with box-and-whisker plots showing median and interquartile range for 7 different gene signatures in the transcriptome-associated clusters: TAC*1 $(n=26), T^{*} 2(n=26), T^{*} 3 a(n=19)$ and $T A C * 3 b(n=33)$. The signatures reflect activation pathways for IL-13 activation of human airway epithelial cells (IL-13 stimulated human bronchial epithelial cells), innate lymphoid cells (ILC1 cells), inflammasome activation, oxidative phosphorylation (OXPHOS), neutrophil activation, lipopolysaccharide (LPS)-stimulated macrophage and interferon- $\gamma$ stimulated macrophage. ${ }^{* * * *} \mathrm{P}<0.0001,{ }^{* * *} \mathrm{P}<0.001,{ }^{* *} \mathrm{P}<0.01,{ }^{*} \mathrm{P}<0.05$. 
(A) PCA Plot

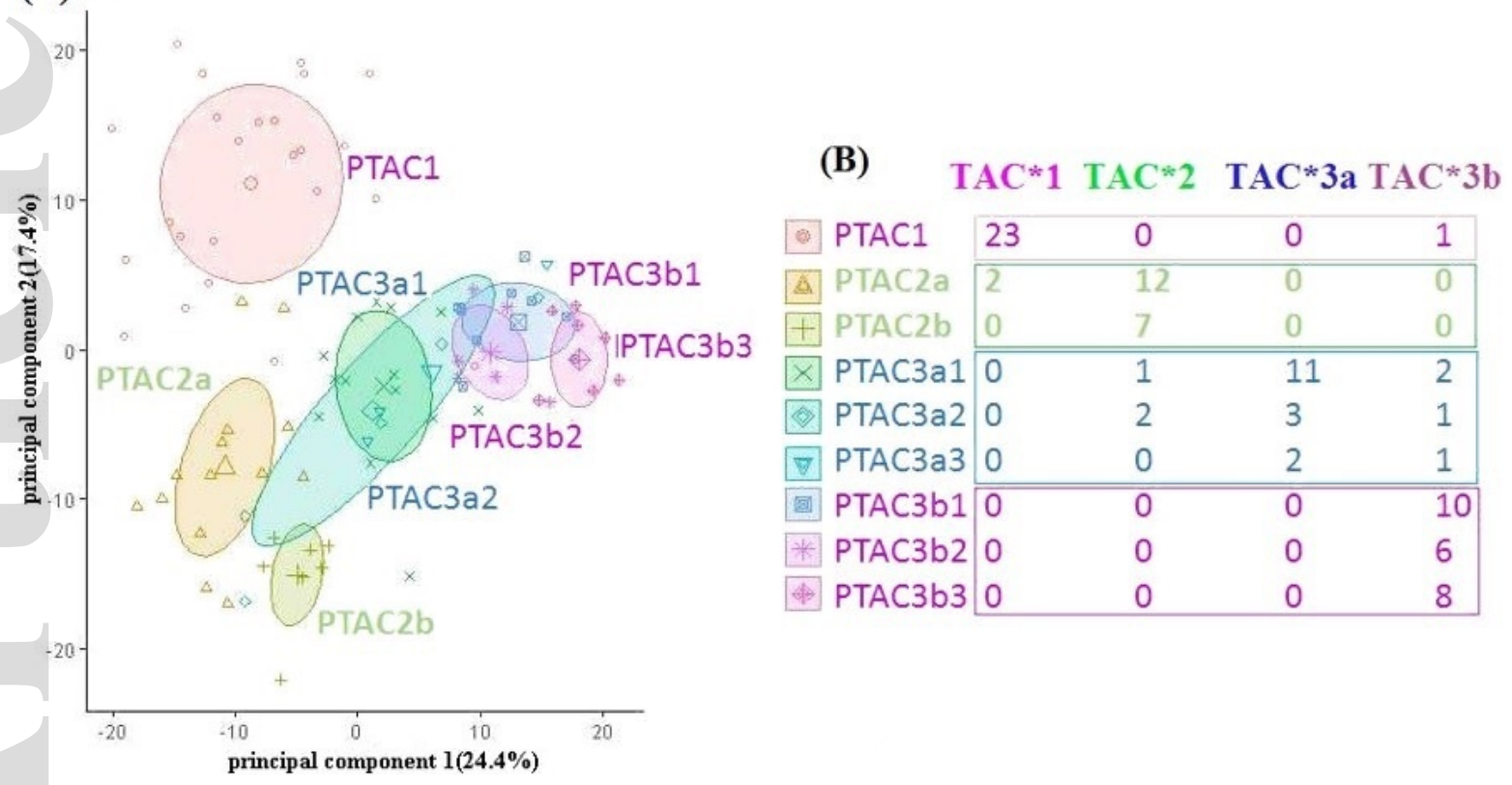

(C)

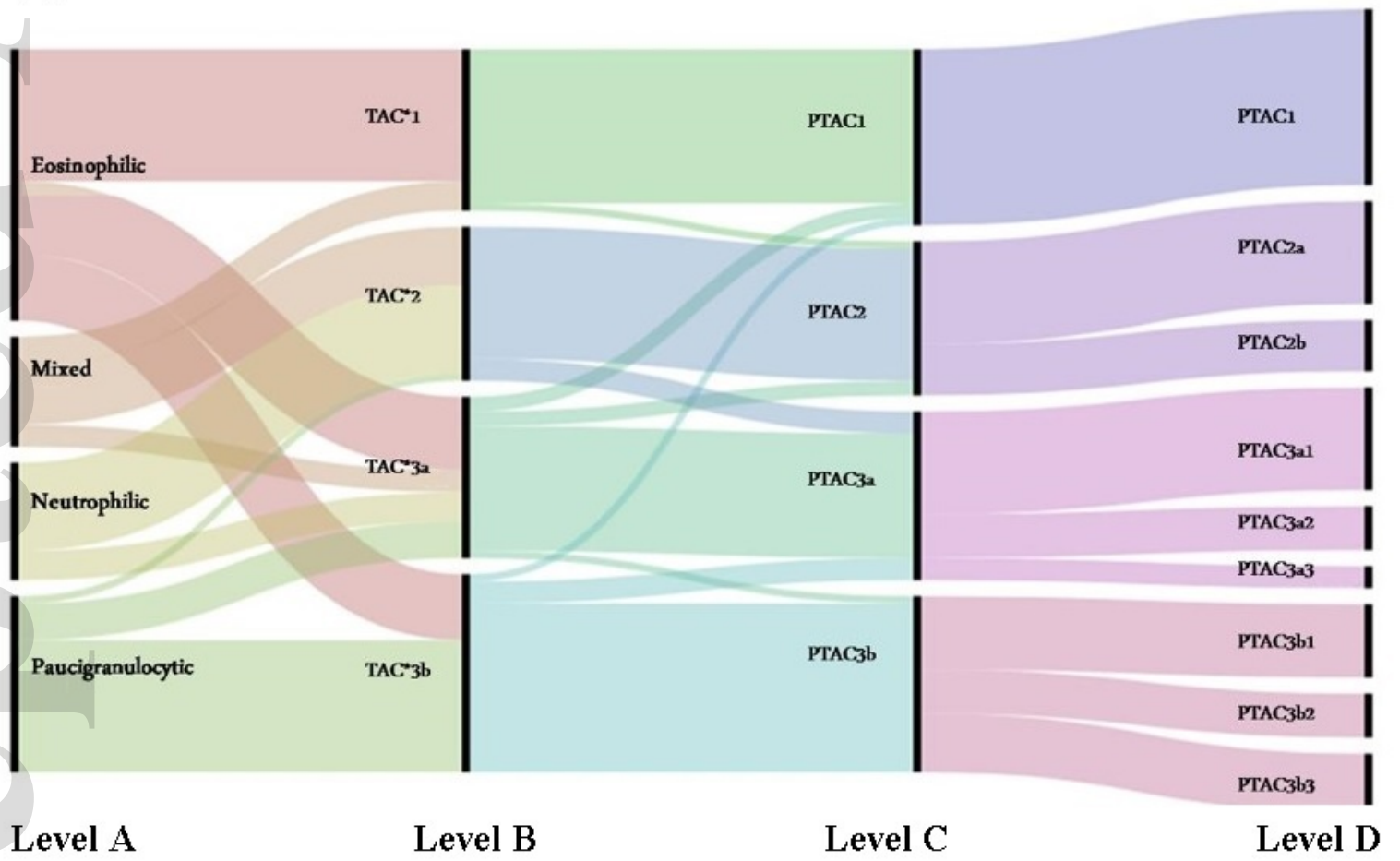

Figure 2. Integration of sputum transcriptomics and serum proteomics. Panel A. Principal component analysis plot identified 9 transcriptomic-associated clusters. The dots represent patients and color code based on cluster subgroups. TAC*1 remains intact shown as PTAC*1; TAC*2 is divided into two subgroups PTAC*2a and PTAC*3b. 
$\mathrm{TAC}^{*} 3 \mathrm{a}$ and $\mathrm{TAC}^{*} 3 \mathrm{~b}$ were divided into 3 subgroups each. Panel $\mathbf{B}$ shows patients in each subgroup. Panel C. Sankey diagram of the directed flow of patients based on the granulocytic state (Level A), transcriptomic-associated clusters (TAC*s) (Level B), transcriptomic- and proteomics-associated clusters (PTACs) (Level C), and PTACs with 9 clusters (Level D). 


\section{References}

1. Kuo CS, Pavlidis S, Loza M, Baribaud F, Rowe A, Pandis I, et al. T-helper cell type 2 (Th2) and nonTh2 molecular phenotypes of asthma using sputum transcriptomics in U-BIOPRED A Transcriptome-driven Analysis of Epithelial Brushings and Bronchial Biopsies to Define Asthma Phenotypes in U-BIOPRED. Eur Respir J. 2017;49(2):443-55.

2. Shaw DE, Sousa AR, Fowler SJ, Fleming L, Roberts G, Corfield J, et al. Clinical and inflammatory characteristics of the European U-BIOPRED adult severe asthma cohort. Eur Respir J. 2015;46(5):1308-21. 3. Tibshirani R, Hastie T, Narasimhan B, Chu G. Diagnosis of multiple cancer types by shrunken centroids of gene expression. Proc Natl Acad Sci U S A. 2002;99(10):6567-72.

4. Hanzelmann S, Castelo R, Guinney J. GSVA: gene set variation analysis for microarray and RNA-seq data. BMC bioinformatics. 2013;14:7.

5. Loza MJ, Djukanovic R, Chung KF, Horowitz D, Ma K, Branigan P, et al. Validated and longitudinally stable asthma phenotypes based on cluster analysis of the ADEPT study. Respiratory research. 2016;17(1):165.

6. Baines KJ, Simpson JL, Wood LG, Scott RJ, Fibbens NL, Powell H, et al. Sputum gene expression signature of 6 biomarkers discriminates asthma inflammatory phenotypes. Journal of allergy and clinical immunology. 2014;133(4):997-1007.

7. Gold L, Ayers D, Bertino J, Bock C, Bock A, Brody EN, et al. Aptamer-based multiplexed proteomic technology for biomarker discovery. PloS one. 2010;5(12):e15004.

8. Burg D, Schofield JPR, Brandsma J, Staykova D, Folisi C, Bansal A, et al. Large-Scale Label-Free Quantitative Mapping of the Sputum Proteome. J Proteome Res. 2018;17(6):2072-91.

9. Wang B, Mezlini AM, Demir F, Fiume M, Tu Z, Brudno M, et al. Similarity network fusion for aggregating data types on a genomic scale. Nature methods. 2014;11(3):333-7.

This article is protected by copyright. All rights reserved 